\title{
Simulation and Analysis of Millimeter-Wave Propagation Characteristics in Complex Office Environment
}

\author{
Yong Li1 ${ }^{1}$ Pengfei Wang2, Feng Chen², Guanyun Wang², Yuanjian Liu² \\ ${ }^{1}$ Zhongxing Limited Corporation of Telecommunications, Shenzhen, China \\ ${ }^{2}$ College of Electronic Science and Engineering, Nanjing University of Posts \& Telecommunications, Nanjing, \\ China \\ Email: 289802167@qq.com
}

Received January 2015

Copyright (C) 2015 by authors and Scientific Research Publishing Inc.

This work is licensed under the Creative Commons Attribution International License (CC BY). http://creativecommons.org/licenses/by/4.0/

(c) (i) Open Access

\begin{abstract}
The several gigabit rate and license-free spectrum resources of $7 \mathrm{GHz}$ bandwidth can be provided by the $60 \mathrm{GHz}$ short-range communication technology, therefore it becomes one of the most promising alternative technologies in the wireless communication. In this paper, the millimeter wave propagation characteristics in the complex office environment are studied by the SBR/Image method. Firstly, under the complex office environment, the propagation characteristics including received power, the arrival angle and the probability distribution of the arrival angle are studied without regard roughness and oxygen absorption loss. Then, the RMS delay spreads in $60 \mathrm{GHz}, 2.4$ $\mathrm{GHz}$ and $5 \mathrm{GHz}$ wireless LAN signals are simulated and compared.
\end{abstract}

\section{Keywords}

Millimeter Wave, Received Power, Arrival Angle, RMS Delay Spread

\section{Introduction}

With the development of intelligent terminal and a rich of wireless data application services, the number of data users increase dramatically and the content of the data is no longer confined to the traditional text or image in the wireless communication system. At the same time, mobile users have more and more demand for high definition video, mobile phone TV and other multimedia services. Wireless network traffic and higher requirements are put forward for the network capacity of the future mobile broadband communications system [1]. According to the description of EU METIS project [2], the goal of the $5 \mathrm{G}$ is that the data traffic increases by 1000 times; 
the rate of user data is raised by 100 times, up to $10 \mathrm{~Gb} / \mathrm{s}$ or more; the number of network devices increases by 100 times; the battery life of low-power devices increases by 10 times and the port-to-port time delay is shortened by 5 times. In the frequency distribution, the $2 \mathrm{G}$ spectrum from $2-6 \mathrm{GHz}$ is selected for $5 \mathrm{G}$, while the UHF band spectrum is selected for indoor scenes coverage. And all functions are completed in the low-cost and sustainable way. The purpose of $5 \mathrm{G}$ is building a network of social. It not only meets the needs of ultra-highspeed transmission, but also satisfies the high capacity, ultra-reliability, flexible communication and other requirements [3].

Recently, the research on the propagation characteristics of the indoor radio wave can be divided into two types, one is the empirical model, and the other is the deterministic model. The deterministic model has two main methods: ray tracing method and finite difference time domain method. Ray tracing methods mainly include mirror method, the minimum optical path method, test-ray method, the deterministic ray tube method and the SBR/image method [4] [5]. The SBR/image method [6] [7] can be used for any complex propagation, and it can find all of radio wave propagation paths from transmitter to receiver and has high computational accuracy and efficiency. The propagation characteristics of $60 \mathrm{GHz}$ millimeter wave in the complex office environment are simulated and analyzed by the SBR/Image method [8].

\section{Environmental Model}

\subsection{Environmental Model}

The specific indoor office under complex environment is modeled to simulate. The $60 \mathrm{GHz}$ signal is selected as simulation signal, and the environmental model is based on the indoor office described in the METIS project file [2]. The planar structure of the indoor office, transmitter location and receiving paths have been shown in Figure 1.

\subsection{Environmental Parameters}

The office environment is composed of the walls, floors, ceilings, screens, desks, etc. Materials of walls, floor and ceiling are concrete, and materials of screens and desks are wood, their corresponding parameters are shown in Table 1, and the materials parameters are shown in Table 2. The size of room is shown in Table 3. The SBR/Image method is used to research and the parameters of SBR/Image method and transmitting and receiving antennas are shown in Table 3.

\section{Simulation Results and Analysis}

\subsection{The Received Power}

The power distribution under indoor office simulation environment is shown in Figure 2. During the simulation, about 4416 receiving points are selected to complete the simulation of received power. From simulation results it can be seen that the received power closest to the transmitting antenna is significantly higher than other locations and the received power around the room is lower. It is suggested that the direct path plays a dominant role

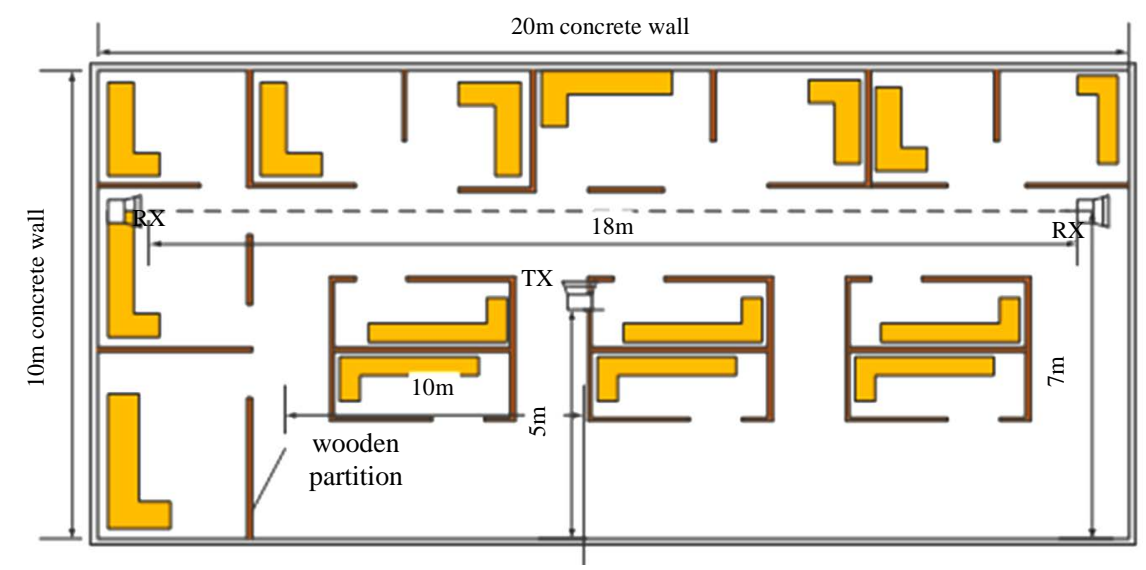

Figure 1. The plan on the complex office. 
Table 1. Materials and height of objects in the office.

\begin{tabular}{ccc}
\hline Object & Height $(\mathrm{m})$ & Material \\
\hline Wall & 2.9 & Concrete \\
Screen & 1.5 & Wood \\
Desk & 0.7 & Wood \\
\hline
\end{tabular}

Table 2. Material parameters.

\begin{tabular}{ccc}
\hline Material & Relative permittivity & Conductivity \\
\hline Concrete & 6.14 & 0.3 \\
Wood & 1.64 & 0.11 \\
\hline
\end{tabular}

Table 3. Simulation environment parameters.

\begin{tabular}{lc}
\hline \multicolumn{1}{c}{ Simulation Environment } & Parameters \\
\hline Length*Width*Height (m) & $20 * 10 * 2.9$ \\
Transmission frequency & $59.5 \mathrm{GHz}$ \\
Transmission power & $10 \mathrm{dBm}$ \\
Transmitting antenna height (m) & 2.8 \\
Receiving antenna height (m) & 1.2 \\
Type of transmitting (receiving) antenna & Omni directional Antenna \\
Polarization of antenna & Vertical polarization \\
Antenna Gain (dBi) & 8.5 \\
\hline
\end{tabular}

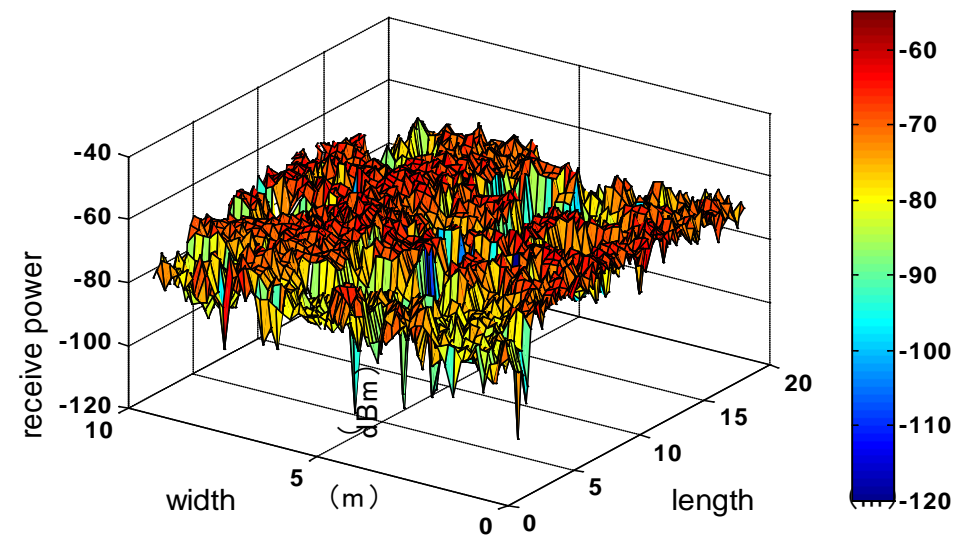

Figure 2. The indoor power distribution.

in the indoor millimeter wave propagation. But the trend of power distribution is ups and downs instead of decreasing from the center to the surrounding and the received power is not completely inversely proportional to the distance from the transmitting antenna, this is because there are reflection and diffraction paths apart from a direct path.

The arrival angles $\bar{\theta}$ and $\bar{\phi}$ as well as their probability distributions are shown in Figure 3(a) and Figure 3(b). The arrival angle $\bar{\phi}$ is the azimuth angle. In this simulation, the direct path is the main path, since the transmitting and receiving antennas are not on the same height. The arriving angle $\bar{\phi}$ distributes around $180^{\circ}$ to $350^{\circ}$ and mainly in the vicinity of $200^{\circ}$ and $340^{\circ}$. The arrival angle $\bar{\theta}$ is the pitch angle and distributes around $53^{\circ}$ to $82^{\circ}$, mainly in the vicinity of $80^{\circ}$. It can be seen that the arrival angles $\bar{\theta}$ and $\bar{\phi}$ don't comply with the probability distribution due to the reflection. 

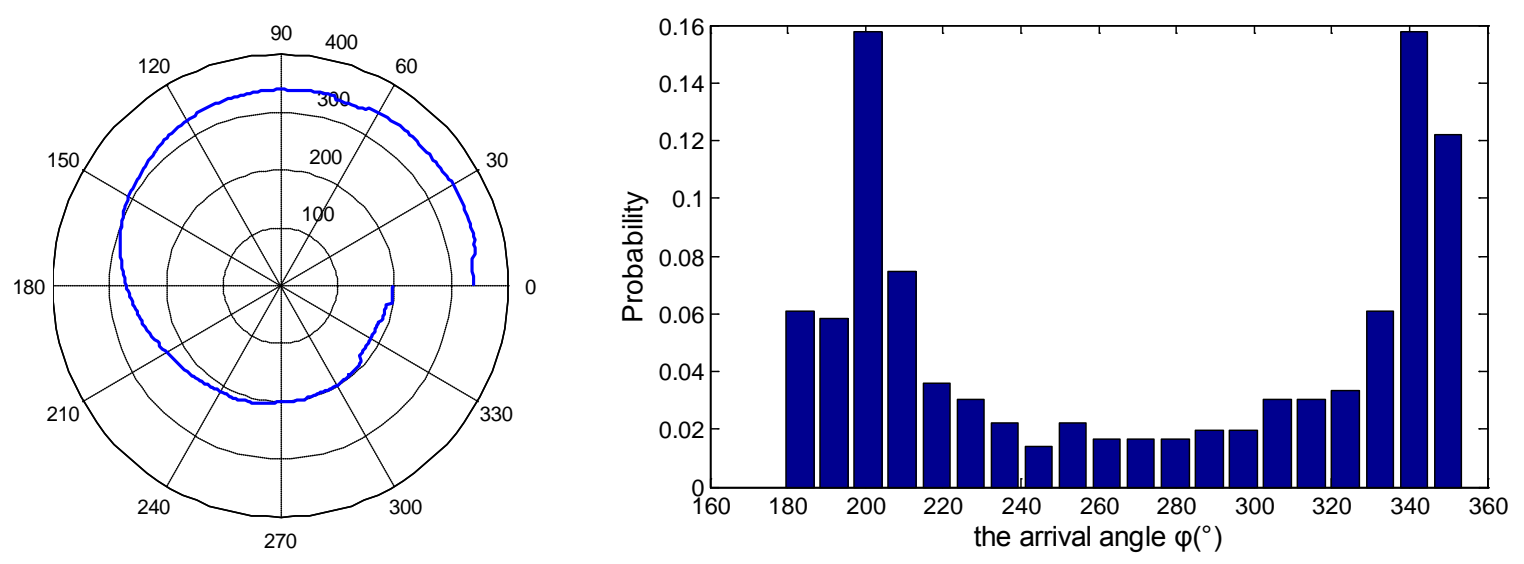

(a)
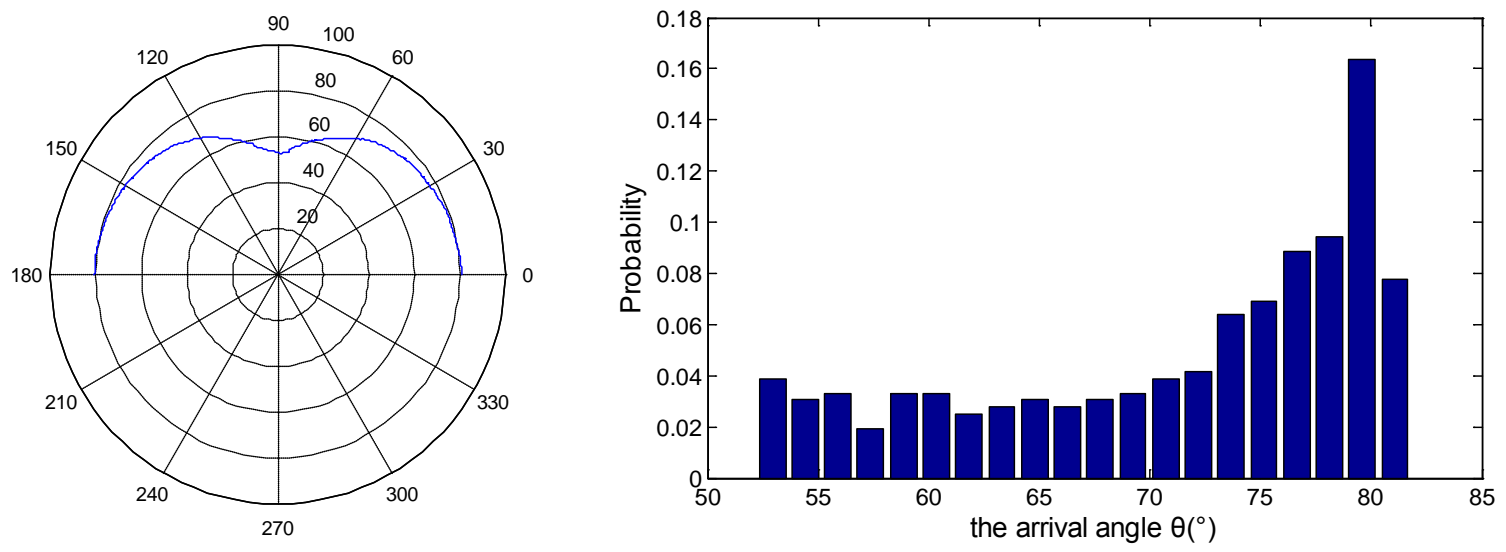

(b)

Figure 3. The arrival angles and their probability distributions. (a) The arrival angle $\bar{\phi}$ and its probability distribution; (b) The arrival angle $\bar{\theta}$ and its probability distribution.

\subsection{The Comparison of RMS Delay Spread in the Different LAN Band}

The RMS delay spread is the second moment of the multipath signal power delay profile. As a good measure of the multipath spread, the RMS delay spread is related to the characteristic deterioration caused by the inter-symbol interference. The comparison among RMS delay spread in $60 \mathrm{GHz}, 2.4 \mathrm{GHz}$ and $5 \mathrm{GHz} \mathrm{LAN}$ signal is shown in Figure 4. According to the simulation results under the office environment, the RMS delay spread in $60 \mathrm{GHz}$ changes among $10.6 \mathrm{~ns}$ to $16.3 \mathrm{~ns}$, the maximum delay occurs at $3 \mathrm{~m}$ distance from the wall, this is because there are many reflection paths caused by the screens, walls, floors and ceilings. However, the minimum delay occurs near the transmitting antenna, because the receiver position is relatively open and there are fewer reflection paths. As can be seen from the figure, the delay spread trends in $60 \mathrm{GHz}, 5 \mathrm{GHz}$ and $2.4 \mathrm{GHz}$ are in agreement, but the value is different, the average of RMS delay spread in $2.4 \mathrm{GHz}$ signal is $14.1 \mathrm{~ns}$, the average of RMS delay spread in $5 \mathrm{GHz}$ signal is $13.5 \mathrm{~ns}$, and the average of RMS delay spread in $60 \mathrm{GHz}$ signal is $13.35 \mathrm{~ns}$, so it can be seen that the RMS delay spread in $60 \mathrm{GHz}$ signal is lower. The smaller the delay spread is, the greater the related bandwidth will be. Therefore, the millimeter wave communication under indoor complex environment can suppress the inter-symbol interference because its delay spread is small.

\section{Conclusion}

In this paper, the SBR/Image method is used to study the problem of millimeter wave propagation under indoor office environment. Through the analysis of simulation results, the millimeter-wave received power under the indoor complex environment mainly comes from the direct path as well as one time and two times reflection 


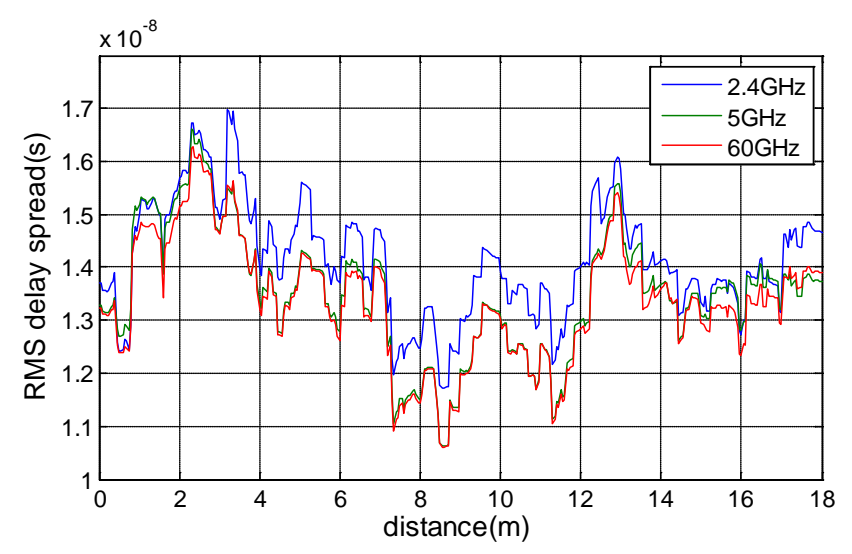

Figure 4. RMS delay spread.

paths caused by the walls and the indoor screen. While the impact of three times reflection is relatively small, three times reflection is still used to simulate, in order to make sure the accuracy and speed of calculation. Moreover, in this simulation, the diffraction and transmission are ignored because they have little impact on the received power. Finally, when applied to the communication under indoor complex environment, the $60 \mathrm{GHz}$ signal has the lower delay spread and more effective suppression of inter-symbol interference than the two other LAN signal. These results can provide a theoretical basis for the planning and design of indoor short-range LAN in the millimeter-wave communication system.

\section{Acknowledgements}

This work has been supported by the ZTE Research Fund.

\section{References}

[1] Lu, Z.-H., Zhang, X.-D., Chen, Y.-J., Xiao, H.H. and Liu, K. (2013) The Analysis of the Future Millimeter-Wave Broadband Mobile Communication System. 43, 12-16.

[2] (2013) METIS Deliverable D6.1 “Simulation Guidelines”. https://www.metis2020.com/

[3] Dong, A.-X. and Wang, X.-J. (2014) 5th Generation Mobile Communication Technology and Development Trends. Communications Technology, 3, 235-240.

[4] Li, Y.-J., Shi, Q.-J. and Zhang, Y.-R. (2013) The Simulation and Analysis of Millimeter-Wave Propagation Characteristics in Indoor Gallery. Microwave Journal, 29, 39-43.

[5] Liu, Y.-J., Shi, Q.-J. and Zhang, Y.-R. (2013) Simulation and Analysis of $60 \mathrm{GHz}$ Millimeter-Wave Indoor Propagation Characteristics Based on the Method of SBR/Image. Progress in Electromagnetic Research, 43, 15-28.

[6] Chen, S.H. and Jeng, S.K. (1996) SBR/Image Approach for Radio Wave Propagation in Tunnels with and without Traffic. IEEE Transactions on Antennas and Propagation, 3, 570-578.

[7] Chen, S.H. (1997) An SBR/Image Approach for Radio Wave Propagation in Indoor Environments with Metallic Furniture. IEEE Transactions on Antennas and Propagation, 1, 98-106. http://dx.doi.org/10.1109/8.554246

[8] Zhang, Q. (2010) Some Issues on 60GHz Millimeter-Wave Short-Range Communication. University of Science and Technology of China. 